\title{
Las transfusiones de sangre podrían aumentan la mortalidad en pacientes con síndrome coronario agudo y anemia
}

Blood transfusions may increase mortality in patients with acute coronary syndrome and anemia

\section{Objetivo}

Comparar la mortalidad total asociada a una estrategia de transfusión de sangre (o uso liberal) versus no transfusión (o uso restringido) en pacientes con síndrome coronario agudo y anemia aguda.

\section{Fuentes de datos}

Búsqueda sistemática de estudios desde enero de 1966 a marzo de 2012 de las siguientes bases de datos: MEDLINE, EMBASE, CINAHL, Scopus, Web of Science, Cochrane.

\section{Selección de estudios}

Se seleccionaron estudios en base a: 1) efectos de la transfusión de sangre o uso liberal versus no transfusión o uso restringido que reportaran mortalidad total y nivel de hemoglobina en los dos grupos, 2) uso de métodos estadísticos para minimizar confundidores.

\section{Resultados Principales}

Se identificaron 203.665 pacientes, provenientes de nueve estudios observacionales y un ensayo clínico controlado y aleatorizado (ECCA). Los resultados se detallan en la tabla 1.

\section{Comentario}

La anemia aguda es un predictor independiente de mala evolución en los pacientes con síndrome coronario agudo'. Existe un delicado balance entre el perjuicio dado por la disminución del aporte de oxigeno con el consecuente empeoramiento de la isquemia que genera la anemia, y la sobrecarga de volumen y el aumento de la trombogenicidad que podría producir la transfusión de sangre. Este meta-análisis evalúa la hipótesis de que la restricción en la transfusión de sangre disminuye la mortalidad total en los pacientes con síndrome coronario agudo y anemia aguda. Los autores concluyen que este esquema se asocia no solo a una reducción en la mortalidad total, sino también a una reducción del IAM. Si bien la hipótesis planteada presenta enorme relevancia clínica y plausibilidad biológica, los resultados de este meta-análisis tienen una validez muy limitada debido a grandes debilidades metodológicas. Nueve de los diez estudios seleccionados fueron observacionales y solo uno fue un ECCA de escasas dimensiones. El criterio de indicación de transfusión fue definido por el médico tratante en cada estudio. Esto es una gran limitación dado que es posible hipotetizar que los pacientes con indicación de transfusión fallecieron más por la gravedad del cuadro clínico (magnitud del sangrado,
Tabla 1

\begin{tabular}{c|c|c|c|c|c} 
& $\begin{array}{c}\text { Uso } \\
\text { liberal }\end{array}$ & $\begin{array}{c}\text { Uso } \\
\text { restringido }\end{array}$ & $\begin{array}{c}\mathbf{R R} \\
\mathbf{( I C 9 5 \% )}\end{array}$ & $\begin{array}{c}\text { NND } \\
(\mathbf{I C 9 5 \% )}\end{array}$ & $\mathbf{p}$ \\
\hline $\begin{array}{c}\text { Mortalidad } \\
\text { total }\end{array}$ & $18,2 \%$ & $10,2 \%$ & $2,91(2,46$ a 3,44$)$ & $12(6$ a 17$)$ & $1<0,001$ \\
\hline
\end{tabular}

RR: riesgo relativo. NND: número necesario a dañar. IC95\%: intervalo de confianza del $95 \%$

En el caso de este estudio los autores calculan una diferencia ponderada de riesgo entre los 2 grupos (12\% de incremento del riesgo absoluto en el grupo liberal).

\section{Conclusiones}

En los pacientes con síndrome coronario agudo y anemia aguda, la estrategia de transfusión de sangre o uso liberal se asoció con un aumento del riesgo de muerte total.

Fuente de financiamiento / Conflicto de intereses: no reportados.

compromiso hemodinámico, sitio del sangrado, etc.), que por el efecto deletéreo de la transfusión en sí misma. Otras limitaciones de trascendencia están relacionadas con la marcada heterogeneidad de los resultados y con el hecho de que no se exploró la relación entre la transfusión de sangre en distintos niveles de hemoglobina para conocer cuál sería el umbral de seguridad de hemoglobina a partir del cual es necesario transfundir.

\section{Conclusiones del comentador}

El presente meta-análisis posee importantes limitaciones metodológicas. Hasta el momento no se puede recomendar una conducta sistemática de uso restringido de transfusión de sangre en estos pacientes. Tan importante como conocer el valor de hemoglobina, es identificar y tratar la causa de sangrado. Para saber cuál es el beneficio neto de la transfusión en los pacientes con síndrome coronario agudo y anemia es imprescindible la realización de un ECCA con adecuado poder y calidad.

Aníbal Arias [ Servicio de Cardiología del Hospital Italiano de Buenos Aires. anibal.arias@ hospitalitaliano.org.ar ]

Arias, A. Las transfusiones de sangre podrían aumentan la mortalidad en pacientes con síndrome coronario agudo y anemia. Evid Act Pract Ambul Oct-Dic 2013; 16(4):132. Comentado de: Chatterjee S, Wetterslev J, Sharma A, et al. Association of blood transfusion with increased mortality in myocardial infarction: a meta-analysis and diversity-adjusted study sequential analysis. JAMA Intern Med. 2013 Jan 28;173(2):132-9. PMID: 23266500.

\section{Referencias}

1. Sabatine M, Morrow D, Giugliano RP y col. Association of Hemoglobin Levels With Clinical Outcomes in Acute Coronary Syndromes. Circulation. 2005;111:2042-2049. 